\title{
Patientin erwacht trotz schlechter Prognose aus dem Koma
}

Manchmal lohnt es sich, Patienten auch in scheinbar hoffnungslosen Situationen nicht aufzugeben: Bei einer 36-jährigen Frau, die bereits seit 20 Tagen nach einem Herzstillstand im Koma lag, deuteten die Befunde auf eine sehr ungünstige Prognose. Dennoch wachte sie einige Tage später wieder auf.

Im Zweifel doch noch einen Versuch wagen - das ist die Botschaft aus einem ungewöhnlichen Verlauf bei einer 36-jährigen Frau, die nach einem Herzstillstand im Koma lag. Die Ärzte sahen eigentlich keine Chance mehr auf eine Erholung, die Angehörigen pochten aber auf eine aggressive Weiterbehandlung. Entgegen aller Wahrscheinlichkeit wachte die Frau nach 29 Tagen tatsächlich wieder auf und gewann langsam ihr Leben zurück.

Die Patientin hatte am Arbeitsplatz einen Herzstillstand erlitten; Mitarbeiter hatten zwar eine Herzdruckmassage versucht, einen stabilen Rhythmus erzielten jedoch erst die Rettungssanitäter nach fünf Defibrillationen und drei Adrenalingaben.

\section{Chance auf Erholung unter 0,5\%}

Nach 20 Tagen Koma untersuchten die Ärzte die kortikalen somatosensibel evozierten Potenziale. Dabei fanden sie bilateral keine negative Abweichung von der isoelektrischen Linie $20 \mathrm{~ms}$ nach Reizung (N20-Antwort). In solchen Fällen liege die Wahrscheinlichkeit für eine günstige Prognose bei weniger als $0,5 \%$, berichten Neurointensivmediziner um Dr. Jessica Weinstein von der Universität in Philadelphia. Sie teilten den Angehörigen daraufhin mit, dass sie letztlich keine Chance für eine bedeutsame neurologische Erholung sähen. Dennoch wollte die Familie der Betroffenen weiterhin eine maximale medizinische Versorgung.

Doch 29 Tage nach dem Herzstillstand öffnete die Patientin völlig überraschend die Augen und schien den Bewegungen des Pflegepersonals zu folgen. Nach weiteren zwei Tagen konnte sie auf Ansprache reagieren. In den folgenden beiden Wochen sahen die Ärzte deutliche kognitive Verbesserungen. Nach 45 Tagen entließen sie die Patientin in die Reha. Zu diesem Zeit- punkt erkannte sie bereits wieder Freunde und Familie und sprach in ganzen Sätzen. Sechs Monate später zeigte sie nur noch eine leichte Dysarthrie und geringe Defizite des Kurzzeitgedächtnisses, auch konnte sie wieder mithilfe eines Stocks gehen.

\section{Kein früher Verzicht auf medizinische Maßnahmen}

Die Intensivmediziner um Weinstein weisen darauf hin, dass bei der Patientin neben der fehlenden N20-Antwort auch ein malignes EEG-Muster bestanden habe. Hinzu komme die lange Komadauer, die ein Erwachen sehr unwahrscheinlich mache. Es hätte wohl kaum jemand für möglich gehalten, dass sich ein Patient unter solchen Umständen wieder er-

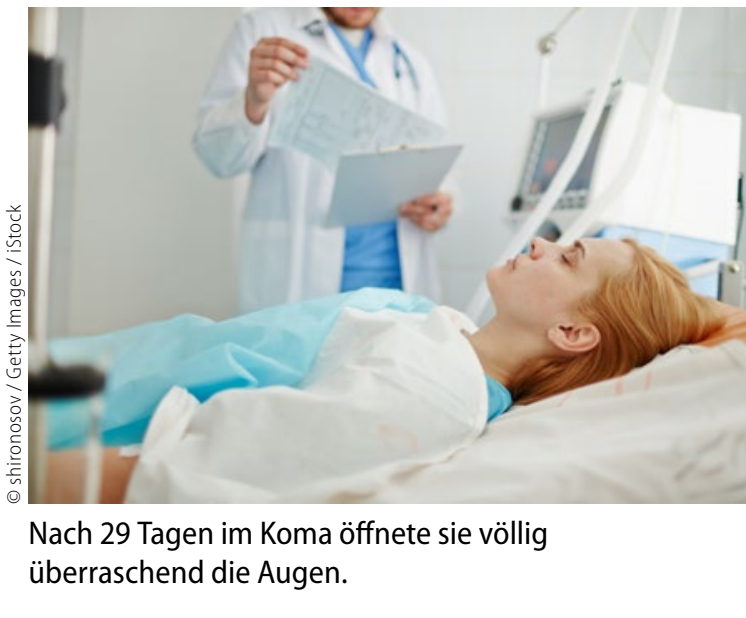

holt. Ein früher Verzicht auf medizinische Maßnahmen wegen vermeintlich schlechter Aussichten sollte unbedingt vermieden werden, schlussfolgern die US-Neurologen.

Thomas Müller

Weinstein J et al. Resuscitation 2017, online 8. Februar; doi: 10.1016/j.resuscitation.2017.01.022

\section{Placebokontrollierte Studie}

\section{Magnesiumoxid hilft doch nicht bei Beinkrämpfen}

\section{Magnesiumoxid verhindert bei Erwachsenen nicht besser als ein Scheinpräparat die Häufigkeit und die Schwere nächtlicher Krämpfe in der Beinmuskulatur.}

Israelische Ärzte haben in einer prospektiven Studie 96 Probanden untersucht, die innerhalb der vergangenen zwei Wochen vier Mal nächtliche Muskelkrämpfe gehabt hatten. 46 Probanden erhielten ein Scheinpräparat, 48 Teilnehmer Magnesiumoxid in einer Dosis von $520 \mathrm{mg}$ an freiem elementarem $\mathrm{Mg}^{2+}$ pro Tag.
Die durchschnittliche Veränderung der Zahl der nächtlichen Beinmuskelkrämpfe betrug -3,41 pro Woche in der Verumgruppe und $-3,03$ pro Woche in der Placebogruppe. Darüber hinaus gab es zwischen den Studiengruppen keinen Unterschied bezüglich der Schwere und der Dauer der Muskelkrämpfe.

Einschränkend weisen die Ärzte darauf hin, dass der Untersuchungszeitraum mit nur vier Wochen kurz war. ple

Maor NR et al. JAMA Intern Med 2017; online 20. Februar. doi: 10.1001/jamainternmed.2016.9261 